\title{
Development and clinical implementation of a new template for MRI-based intracavitary/interstitial gynecologic brachytherapy for locally advanced cervical cancer: from CT-based MUPIT to the MRI compatible Template Benidorm. Ten years of experience
}

\author{
Silvia Rodriguez Villalba, MD, PhD', Jose Richart Sancho, MScl, Antonio Otal Palacin, MSc', Jose Perez-Calatayud, PhDl, 2 , \\ Manuel Santos Ortega, PhD' \\ IRadiotherapy Department, Hospital Clínica Benidorm, Benidorm, Alicante, ${ }^{2}$ Radiotherapy Department, La Fe University and Polytechnic \\ Hospital, Valencia, Spain
}

\begin{abstract}
Purpose: To study outcome and toxicity in 59 patients with locally advanced cervix carcinoma treated with computed tomography (CT)-based Martinez universal perineal interstitial template (MUPIT) and the new magnetic resonance imaging (MRI)-compatible template Benidorm (TB).

Material and methods: From December 2005 to October 2015, we retrospectively analyzed 34 patients treated with MUPIT and 25 treated with the TB. Six 4 Gy fractions were prescribed to the clinical target volume (CTV) combined with external beam radiotherapy (EBRT). The organs at risk (OARs) and the CTV were delineated by CT scan in the MUPIT implants and by MRI in the TB implants. Dosimetry was CT-based for MUPIT and exclusively MRI-based for TB. Dose values were biologically normalized to equivalent doses in $2 \mathrm{~Gy}$ fractions $\left(\mathrm{EQD}_{2}\right)$.

Results: Median CTV volumes were $163.5 \mathrm{~cm}^{3}$ for CT-based MUPIT (range 81.8-329.4 $\mathrm{cm}^{3}$ ) and $91.9 \mathrm{~cm}^{3}$ for MRIbased TB (range 26.2-161 $\mathrm{cm}^{3}$ ). Median $\mathrm{D}_{90}$ CTV (EBRT + BT) was 75.8 Gy for CT-based MUPIT (range 69-82 Gy) and 78.6 Gy for MRI-based TB (range 62.5-84.2 Gy). Median $\mathrm{D}_{2 \mathrm{~cm}^{3}}$ for the rectum was $75.3 \mathrm{~Gy}$ for CT-based MUPIT (range 69.8-132.1 Gy) and 69.9 Gy for MRI-based TB (range 58.3-83.7 Gy). Median $\mathrm{D}_{2 \mathrm{~cm}^{3}}$ for the bladder was 79.8 Gy for CT-based MUPIT (range 71.2-121.1 Gy) and 77.1 Gy for MRI-based TB (range 60.5-90.8 Gy). Local control (LC) was $88 \%$. Overall survival (OS), disease free survival (DFS), and LC were not statistically significant in either group. Patients treated with CT-based MUPIT had a significantly higher percentage of rectal bleeding G3 $(p=0.040)$ than those treated with MRI-based TB, $13 \%$ vs. $2 \%$.

Conclusions: Template Benidorm treatment using MRI-based dosimetry provides advantages of MRI volume definition, and allows definition of smaller volumes that result in statistically significant decreased rectal toxicity compared to that seen with CT-based MUPIT treatment.
\end{abstract}

Key words: cervical cancer, CT, dosimetry, endocavitary, interstitial, MRI.

\section{Purpose}

Perineal interstitial brachytherapy is used in locally advanced cervix carcinoma $[1,2,3,4,5,6,7,8]$. The current commercially available interstitial templates are: Martinez universal perineal interstitial template (MUPIT) (Nucletron, Elekta AB, Stockholm, Sweden) [9] and the Syed template (Best Medical International, Inc. Springfield, VA, USA) [10]. Their standard commercial version does not have an intracavitary component (IC) and they are (at least
MUPIT template) incompatible with magnetic resonance imaging (MRI), thus necessitating the use of computed tomography (CT) dosimetry. Both have the possibility of adding an intracavitary component, but do not fit perfectly. In addition, Syed template can be used with MRI with titanium needles.

Until April of 2013, we employed MUPIT as a part of radical treatment. In order to improve perineal interstitial brachytherapy by incorporating MRI into these treatments, we developed Template Benidorm (TB) [11,12] (Lor-
Address for correspondence: Silvia Rodriguez Villalba, MD, PhD, Radiotherapy Department, Hospital Clinica Benidorm, Avenida Alfonso Puchades 8, 03501 Benidorm, Alicante, Spain, phone: +34 606557346, +34 966072725, fax: +34 9966072724, « e-mail: srodriguez@clinicabenidorm.com
Received: 14.05 .2016 Accepted: 22.08.2016 Published: 31.10.2016 
ca Marín S.A, Murcia, Spain). This applicator is completely compatible with MRI and allows for combination of intracavitary radiotherapy and MRI-compatible transperineal interstitial needles (IS). In 2007, with the incorporation of MRI in our department for brachytherapy treatments of gynecologic tumors, we have begun to follow the recommendations of ABS/GEC-ESTRO for volume definitions and dose-volume analysis $[13,14]$ for image guided brachytherapy (IGBT).

The aim of this study is to retrospectively assess treatment outcome of 59 patients with primary, locally advanced cervix carcinoma, who were treated with perineal interstitial brachytherapy with MUPIT (34 patients) and IC/IS brachytherapy with TB (25 patients), and to analyze potential differences in dosimetry, clinical results, and toxicity in both groups of patients, with the main difference of possibility to incorporate MRI in volume definitions and dose-volume dosimetry with TB.

\section{Material and methods}

From December 2005 to October 2015, 108 patients with malignant cervical tumors were treated with perineal interstitial brachytherapy. We excluded from this analysis 28 vaginal vault or cervical stump recurrences, and 21 patients without follow-up. The remaining 59 patients with histologically proven primary carcinoma of the cervix were treated: 34 with MUPIT and, after April 2013, 25 patients were treated with TB (Table 1 ).

The developed template adapts the currently existing manufactured MRI compatibles of intrauterine tubes (Nucletron, Elekta). These 15, 30, and 45 grade tubes are 4,6 , and $8 \mathrm{~cm}$ long, allowing the deliver of a large central dose. This device can also use 20 or $24 \mathrm{~cm}$ long titanium needles to cover the disease in all directions. The intracavitary component helps to straighten the uterus, while maintaining the straight geometry of the parallel and angled needles. The TB has 12 rows, $1.1 \mathrm{~cm}$ apart (measured from center to center of each needle), in order to introduce straight and angled titanium needles. It has 7 rows, in which there are 35 straight holes for parallel needle placement, and 5 rows, in which there are 16 angled holes $\left(7^{\circ}\right)$. This template allows coverage of the distal parametrium (up to $4.5 \mathrm{~cm}$ from the middle of the intracavitary tube) and the entire vagina. It has also the possibility to include a vaginal cylinder $2.4 \mathrm{~cm}$ in diameter; these cylinders are available in lengths from $4,6,8,10$, and $13 \mathrm{~cm}$ to accommodate different vaginal lengths, with 8 positions of needles in its surface. Plastic obturator tracks fasten the needles to avoid displacement. A second plate of the exactly same design, covers the first in order to achieve adequate docking and to prevent displacement of the components of the applicator. This second plate can hold a central needle when the introduction of the intrauterine tube is not possible (Figure 1).

Brachytherapy treatment planning was done one week before the procedure. Pre-implant imaging with CT (LightSpeed VCT, GE Medical Systems, Milwaukee, Wisconsin, USA) for MUPIT patients, or MRI (Optima MR450w, GE Medical Systems, Milwaukee, Wisconsin,
USA) for TB patients was completed with the respective templates and a vaginal obturator of a known length (13 $\mathrm{cm}$ for MUPIT and $4-12 \mathrm{~cm}$ for TB according to the vaginal length) in place, and the bladder filled with $50 \mathrm{~cm}^{3}$ of saline solution. Contiguous $2.5 \mathrm{~mm}$ thick CT slices and T2 weighted axial MRI images were taken (Figure 2A-F) [12]. The number, position, and depths of the needles were defined using these images, always allowing an offset of $10 \mathrm{~mm}$ from the tip of the needle to describe the first dwell position. Thus, the depth was considered appropriate if

Table 1. Patients and disease characteristics

\begin{tabular}{|c|c|c|c|}
\hline Factors & Total & MUPIT $(n=34)$ & TB $(n=25)$ \\
\hline Age & & $54.5 \mathrm{yr}(36-79)$ & $57 \mathrm{yr}(33-77)$ \\
\hline \multicolumn{4}{|l|}{$T^{*}$} \\
\hline $\mathrm{T} 2 \mathrm{~A}$ & $1(2 \%)$ & - & $1(2 \%)$ \\
\hline $\mathrm{T} 2 \mathrm{~B}$ & $29(49 \%)$ & 17 (29\%) & $12(20 \%)$ \\
\hline T3A & $3(5 \%)$ & $2(3 \%)$ & $1(2 \%)$ \\
\hline $\mathrm{T} 3 \mathrm{~B}$ & $15(25 \%)$ & $10(17 \%)$ & $5(8 \%)$ \\
\hline $\mathrm{T} 4$ & 11 (19\%) & $5(8 \%)$ & $6(10 \%)$ \\
\hline \multicolumn{4}{|l|}{$\mathrm{N}^{*}$} \\
\hline NO & 45 (76\%) & 29 (49\%) & $16(27 \%)$ \\
\hline N1 & 11 (19\%) & $5(8 \%)$ & $6(10 \%)$ \\
\hline M1 & $3(5 \%)$ & - & $3(5 \%)$ \\
\hline \multicolumn{4}{|l|}{ Stage } \\
\hline$\| \mathrm{A}$ & $1(2 \%)$ & - & $1(2 \%)$ \\
\hline$\| B$ & $27(46 \%)$ & 17 (29\%) & $10(17 \%)$ \\
\hline IIIA & $3(5 \%)$ & $2(3 \%)$ & $1(2 \%)$ \\
\hline IIIB & $14(24 \%)$ & $10(17 \%)$ & $4(7 \%)$ \\
\hline $\operatorname{IVA}^{\star \star \star}$ & $11(18 \%)$ & $5(8 \%)$ & $6(10 \%)$ \\
\hline$I V B^{\star \star \star \star}$ & $3(5 \%)$ & - & $3(5 \%)$ \\
\hline
\end{tabular}

\begin{tabular}{llll}
\hline \multicolumn{4}{l}{ Vaginal involvement } \\
\hline No & $19(32 \%)$ & $10(17 \%)$ & $9(15 \%)$ \\
\hline Yes & $40(68 \%)$ & $24(41 \%)$ & $16(27 \%)$
\end{tabular}

Parametrial involvement

\begin{tabular}{lccc}
\hline Free & $7(12 \%)$ & $4(7 \%)$ & $3(5 \%)$ \\
\hline Unilateral & $21(36 \%)$ & $15(25 \%)$ & $6(10 \%)$ \\
\hline Bilateral & $31(52 \%)$ & $15(25 \%)$ & $16(27 \%)$ \\
\hline $\begin{array}{l}\text { Myometrial involvement } \\
\text { Yes }\end{array}$ & $15(25 \%)$ & $8(14 \%)$ & $7(12 \%)$ \\
\hline No & $44(75 \%)$ & $26(44 \%)$ & $18(31 \%)$
\end{tabular}

*AJCC tumor node metastasis (TNM) [16]

**International Federation of Gynecology and Obstetrics (FIGO 2009) [15] ***/nfiltration of bladder [6] or rectum [5]

****Para-aortic lymph node metastases [2] or supraclavicular metastases [1] 

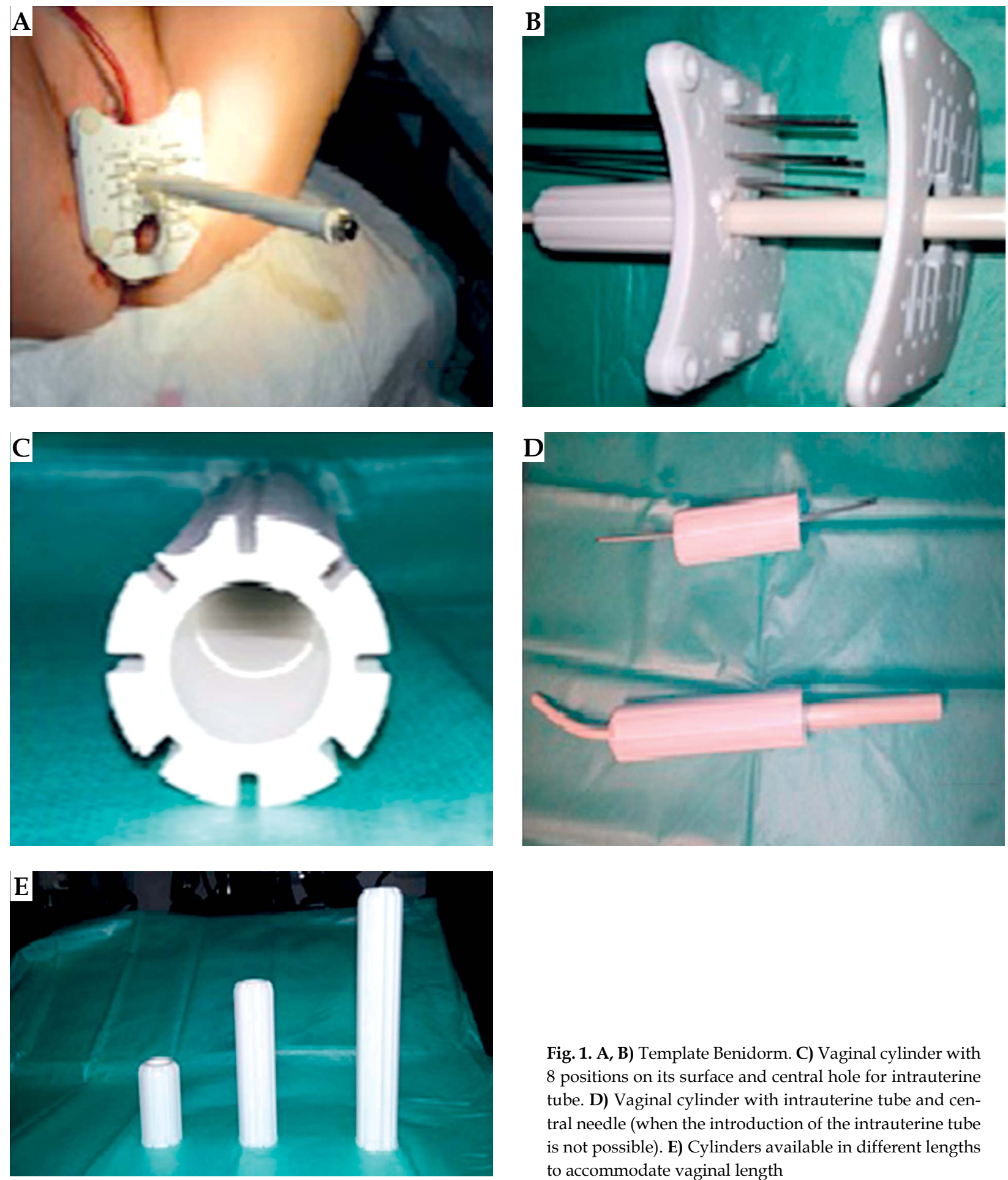

Fig. 1. A, B) Template Benidorm. C) Vaginal cylinder with 8 positions on its surface and central hole for intrauterine tube. D) Vaginal cylinder with intrauterine tube and central needle (when the introduction of the intrauterine tube is not possible). E) Cylinders available in different lengths to accommodate vaginal length

the clinical target volume (CTV) was entirely covered up to a $10 \mathrm{~mm}$ superior margin.

We added TB to the applicator library of treatment planning system (TPS), so that the system could prepare a dosimetrically accurate pre-plan that would enhance the safety of the procedure (Figure 2G, H) [17]. Although this pre-plan was done without an intrauterine tandem, the interstitial component was adapted to the CTV for an optimal procedure, mainly in cases in which extensive

disease had so distorted the anatomy that adequate coverage of the CTV would have been difficult to achieve.

Implantation of the applicator was similar with either MUPIT or TB [11]. Median number of needles employed was 17 (range 12-23 needles) in MUPIT, and 16 in TB (range 10-19 needles).

Computed tomography was used for dosimetry for MUPIT patients, and a $1.5 \mathrm{~T}$-axial MRI sequence (T2 weighted fast recovery fast spin echo [FRFSE]) was used 
for TB patients (Figure 3A, B). Normal saline solution $\left(50 \mathrm{~cm}^{3}\right)$ was injected into the bladder to help in volume definition. Hyoscine-n-butylbromide $(20 \mathrm{mg})$ was administrated intravenously for aperistaltic effect to TB patients before MRI.

Six 4 Gy fractions were prescribed to CTV over four days, six hours apart. Due to the dose characteristics in- volved in using a single interstitial implant and a single prescription dose over the CTV, the organs at risk (OARs) (rectum and bladder) and the CTV were delineated in the CT scan in MUPIT implants and in the MRI in TB implants. In every case, the same radiation oncologist, with more than 15 years of experience in interstitial brachytherapy, performed this function. With either template, the CTV
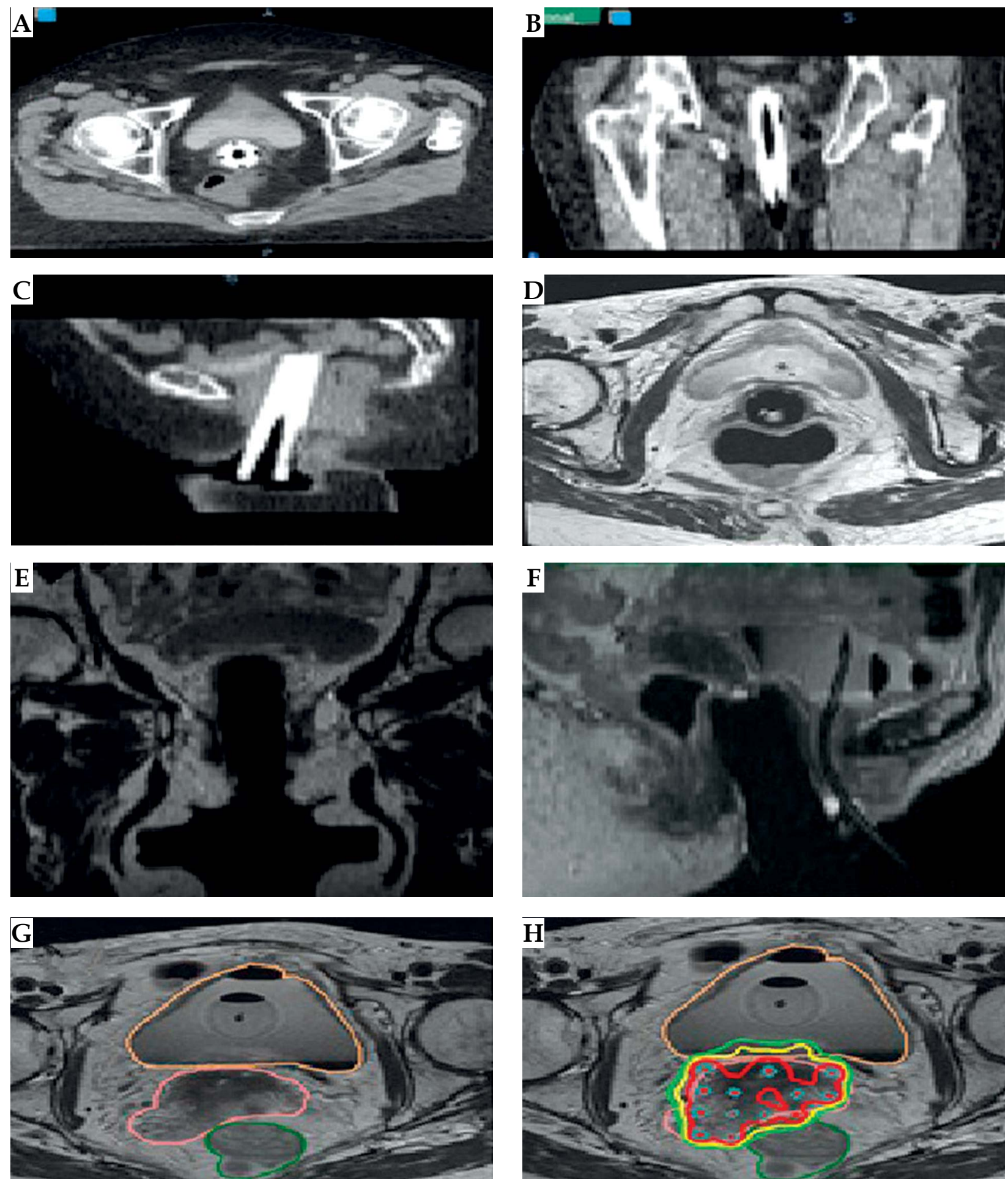

Fig. 2. A-C) Computed tomography for pre-plan in MUPIT. D-F) Magnetic resonance imaging (MRI) for pre-plan in Template Benidorm (TB). G, H) Pre-plan MRI (Library) for TB 

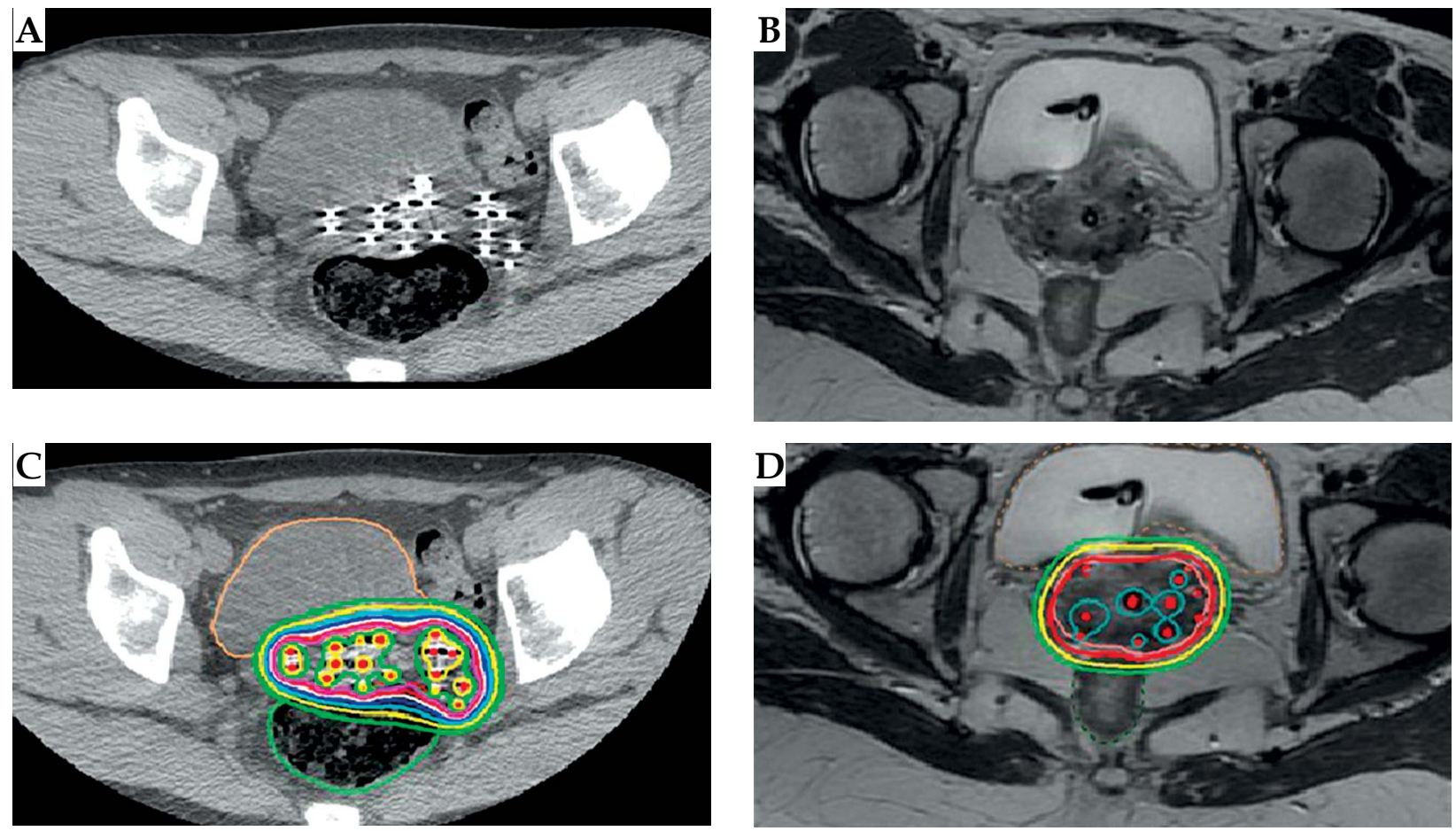

Fig. 3. A) Planning computed tomography in MUPIT. B) Planning magnetic resonance imaging in Template Benidorm (TB).

C) Computed tomography-based dosimetry in MUPIT. D) Magnetic resonance imaging-based dosimetry in TB

was defined taking into account initial imaging studies, clinical examination at the first consultation, and direct examination under anesthesia at the time of the implant. Gross tumor volume (GTV) at diagnosis and GTV at the time of brachytherapy were unified in a single CTV (that includes GTV, CTV $\mathrm{HR}^{\prime}$ and $\mathrm{CTV}_{\mathrm{IR}}$ ), based on GEC-ESTRO recommendations and ICRU $89[5,18,19,20]$.

The dosimetry was optimized to improve the implant coverage and to reduce rectal/bladder doses. Dose constraints applied to the OAR were based on the ABS/ GEC-ESTRO that recommended maximal dose of 75 Gy $\left(\mathrm{EQD}_{2}\right)$ to $2 \mathrm{~cm}^{3}$ of the rectum and sigmoid, and $85 \mathrm{~Gy}$ $\left(E Q D_{2}\right)$ to the $2 \mathrm{~cm}^{3}$ of the bladder. The cumulative equivalent dose (external beam radiotherapy [EBRT] and brachytherapy) for $2 \mathrm{~Gy}\left(\mathrm{EQD}_{2}\right)$ for OARs was calculated assuming $\alpha / \beta$ of 10 for the $C T V$, and 3 for OARs as recommended [13,14] (Figure 3C, D).

Seven patients treated with TB were scanned with both CT and MRI. Clinical target volumes and OARs were defined in both image sets by the same practitioner to develop both CT-based and MRI-based treatment plans. Volumes and DVH-related parameters were compared.

All statistical analysis was done with SPSS 15.0 software. Actuarial survivals were estimated using KaplanMeier analysis. The impact of various prognostic factors on survival was evaluated using the log rank test with a significance threshold of $p \leq 0.05$.

\section{Results}

The median age was 56 years (range 33-79 years). There were 50 squamous cell carcinomas (85\%), 5 adenocarcinomas $(9 \%), 2$ neuroendocrine tumors $(3 \%)$, and
2 papillary squamous cell carcinomas (3\%). Magnetic resonance imaging was performed for diagnosis in all patients. The patients were clinically and radiologically (MRI) staged [15]. To compare data of topography and dimensions in the treatments with each applicator and any potential relationship with local control or toxicity, we have also determined if there were vaginal extension. Additionally, we have noted whether any parametrial involvement was free, unilateral, or bilateral, or any myometrial involvement. Patients and disease characteristics are shown in Table 1.

All patients were treated with a combination of EBRT to the pelvis or to the pelvis and para-aortic (N1 and M1 patients; $n=10,17 \%)$. Thirteen patients $(22 \%)$ received radiotherapy with a lymph node boost (LNB), and 6 patients $(10 \%)$ received a parametrial boost (PMB). The median EBRT dose was 50 Gy (range 45-55 Gy), the median LNB was 10.8 Gy (range 8.4-16.2 Gy), and the median PMB was 5 Gy (range 5-10 Gy). All patients received weekly, concomitant, cisplatin-based chemotherapy. After EBRT, interstitial brachytherapy was applied.

After EBRT (43 patients, 73\%) and before brachytherapy, radiological response and anatomic changes were evaluated using MRI. In eleven patients, there was a radiologic complete response, and in 32 there was a partial response. Fifty-nine patients with primary locally advanced cervix carcinoma, 34 treated with MUPIT and 25 with TB, were analyzed. Median CTV volumes and relationship to the tumor extension defined with CT (MUPIT) and MRI (TB) are given in Table 2. Median $\mathrm{D}_{90}$ of the CTV, median rectum $D_{2 \mathrm{~cm}^{3}}$, and median bladder $\mathrm{D}_{2 \mathrm{~cm}^{3}}$ with both applicators are shown in Table 3. The dosimetric results for 7 patients with volumes defined using both 
Table 2. Median clinical target volumes according to stage

\begin{tabular}{lcc} 
Factor & MUPIT $(n=34)$ & TB $(n=25)$ \\
\hline $\begin{array}{l}\text { Total } \\
\text { (range) }\end{array}$ & $\begin{array}{c}163.5 \mathrm{~cm}^{3}( \pm 59.09) \\
\left(81.8-329.4 \mathrm{~cm}^{3}\right)\end{array}$ & $\begin{array}{c}91.9 \mathrm{~cm}^{3}( \pm 32.57) \\
\left(26.2-161 \mathrm{~cm}^{3}\right)\end{array}$ \\
\hline Tumor stage & & \\
\hline T2A & - & $101.8 \mathrm{~cm}^{3}$ \\
\hline T2B & $139.6 \mathrm{~cm}^{3}$ & $85.8 \mathrm{~cm}^{3}$ \\
& $\left(81.8-241.2 \mathrm{~cm}^{3}\right)$ & $\left(26.2-123 \mathrm{~cm}^{3}\right)$ \\
\hline T3A & $212.4 \mathrm{~cm}^{3}$ & $83.3 \mathrm{~cm}^{3}$ \\
& $\left(158.2-266.6 \mathrm{~cm}^{3}\right)$ & \\
\hline T3B & $214.8 \mathrm{~cm}^{3}$ & $75.3 \mathrm{~cm}^{3}$ \\
& $\left(155.6-329.4 \mathrm{~cm}^{3}\right)$ & $\left(59.8-161 \mathrm{~cm}^{3}\right)$ \\
\hline T4A & $157.4 \mathrm{~cm}^{3}$ & $110.7 \mathrm{~cm}^{3}$ \\
& $\left(135.8-198 \mathrm{~cm}^{3}\right)$ & $\left(79.4-147.7 \mathrm{~cm}^{3}\right)$
\end{tabular}

MRI and CT for the same implant are shown in Table 4. Median follow-up was 25 months (3-101 months).

Overall survival (OS) was $87 \%, 64 \%$, and $53 \%$ at 12,24 , and 36 months, respectively. Disease free survival (DFS) was $89 \%, 70 \%$, and $60 \%$ at 12,24 , and 36 months, respectively. Twenty-two patients (37\%) died because of tumor, 30 are alive without tumor $(51 \%), 4$ patients are alive with tumor $(7 \%)$, and 3 have died of other causes $(5 \%)$. There is not statistical significance in overall survival $(p=0.948)$, disease free survival $(p=0.785)$, local control $(p=0.924)$, lymph node control $(p=0.962)$, and distant failure $(p=0.387)$ in patients treated with MUPIT or TB.

The lymph node failure (LNF, pelvic or para-aortic) rate was $25 \%$, and $27 \%$ developed distant metastases (DM). Median time of lymph node recurrence was 9 months (3-25 months). Median time of distant failure was 19 months (5-40 months).

Thirteen patients (9 MUPIT and 4 TB) experienced local recurrence $(22 \%)$ (in cervix, vagina, parametrium, or uterus) at a median time of 12 months after BT treatment (12-27 months). Five of these $(39 \%)$ had recurrence in the myometrium (4 MUPIT/1 TB). Twelve of 13 local recurrences $(92 \%)$ had a partial response after EBRT plus chemotherapy. Median CTV $\mathrm{D}_{90}$ of the recurrences was 74 Gy (70.8-79.2 Gy) in CT-based MUPIT, and 79.2 Gy (71.7-84.2 Gy) in MRI-based TB.

Toxicity was assessed according to National Cancer Institute CTCAE recommendations (version 4.0). Chronic toxicities are defined at those occurring after $\geq 3$ months of finishing the last brachytherapy procedure.

\section{Acute toxicity}

Two $(3 \%)$ patients had a cervix hemorrhage when the implant was removed (1 MUPIT, $1 \mathrm{~TB}$ ), needing transfusion. There were no G3 acute GU or GI toxicities in these group.

\section{Chronic toxicity}

Four patients $(7 \%), 2$ with stage IIIB disease and 2 with stage IVA, developed a recto-vaginal fistula (3 CTbased MUPIT and 1 MRI-based TB). Two of these were
Table 3. Median $\mathrm{D}_{90}$ of clinical target volumes and $\mathrm{D}_{2 \mathrm{~cm}^{3}}$ of rectum and bladder $\left(E Q D_{2}, E B R T+H D R\right)$ in MUPIT and Template Benidorm

\begin{tabular}{ccc} 
& MUPIT $(n=34)$ & TB $(n=25)$ \\
\hline $\mathrm{D}_{90}$ CTV & $75.8 \mathrm{~Gy}( \pm 3.29)$ & $78.6 \mathrm{~Gy}( \pm 5.88)$ \\
\hline Range & $69-82 \mathrm{~Gy}$ & $62.5-84.2 \mathrm{~Gy}$ \\
\hline $\mathrm{D}_{2 \mathrm{~cm}^{3} \text { rectum }}$ & $75.3 \mathrm{~Gy}( \pm 11.52)$ & $69.9 \mathrm{~Gy}( \pm 6.48)$ \\
\hline Range & $69.8-132.1 \mathrm{~Gy}$ & $58.3-83.7 \mathrm{~Gy}$ \\
\hline $\mathrm{D}_{2 \mathrm{~cm}^{3} \text { bladder }}$ & $79.8 \mathrm{~Gy}( \pm 13.61)$ & $77.1 \mathrm{~Gy}( \pm 8.55)$ \\
\hline Range & $71.2-123.1 \mathrm{~Gy}$ & $60.5-90.8 \mathrm{~Gy}$
\end{tabular}

$E Q D_{2}$ - equivalent dose at $2 \mathrm{~Gy}$; EBRT - external beam radiotherapy, HDR - high dose-rate, $D_{90}$ - the minimum dose received by $90 \%$ of the prostate volume, $C T V$-clinical target volume, $D_{2 \mathrm{~cm}^{3}}$ - minimum dose to the most exposed $2 \mathrm{~cm}^{3}$

Table 4. Median volumes of clinical target volumes (CTV), $D_{90}$ CTV and $D_{2 \mathrm{~cm}^{3}}$ of rectum and bladder $\left(E Q D_{2}\right.$, EBRT + HDR) in 7 patients in computed tomography and magnetic resonance imaging, treated with Template Benidorm for the same implant

\begin{tabular}{lcc} 
& MRI TB & CT TB \\
\hline Volume $\mathrm{cm}^{3}$ & $109.4 \mathrm{~cm}^{3}( \pm 32.86)$ & $135.5 \mathrm{~cm}^{3}( \pm 29.09)$ \\
& $\left(74.1-161 \mathrm{~cm}^{3}\right)$ & $\left(114-184.7 \mathrm{~cm}^{3}\right)$ \\
\hline $\mathrm{D}_{90} \mathrm{CTV}$ & $76.3 \mathrm{~Gy}( \pm 3.84)$ & $76.2 \mathrm{~Gy}( \pm 2.72)$ \\
& $(71.5-80.9 \mathrm{~Gy})$ & $(72.5-79.9 \mathrm{~Gy})$ \\
\hline $\mathrm{D}_{2 \mathrm{~cm}^{3}}$ rectum & $74.8 \mathrm{~Gy}( \pm 5.91)$ & $76 \mathrm{~Gy} \pm$ 3.14) \\
& $(63.3-79.9 \mathrm{~Gy})$ & $(70.2-79.8 \mathrm{~Gy})$ \\
\hline $\mathrm{D}_{2 \mathrm{~cm}^{3}}$ bladder & $75.8 \mathrm{~Gy}( \pm 8.91)$ & $79.2 \mathrm{~Gy}( \pm 4.18)$ \\
& $(61.6-86 \mathrm{~Gy})$ & $(74-85.1 \mathrm{~Gy})$
\end{tabular}

$E Q D_{2}$ - equivalent dose at $2 G y, E B R T$ - external beam radiotherapy, $H D R$ high-dose-rate, MRI - magnetic resonance imaging, TB - Template Benidorm, CT - computed tomography, $D_{90}$ - the minimum dose received by $90 \%$ of the prostate volume, CTV - clinical target volume, $D_{2 \mathrm{~cm}^{3}}$ - minimum dose to the most exposed $2 \mathrm{~cm}^{3}$

associated with local recurrence. One patient with stage T3B disease treated with CT-based MUPIT developed G4 cystitis, 29 months after brachytherapy; this condition was resolved with hyperbaric oxygen. In this case, the CTV $\mathrm{D}_{90}$ was 78.8 , but the bladder $\mathrm{D}_{2 \mathrm{~cm}^{3}}$ was $103.7 \mathrm{~Gy}$.

Eighteen patients (30\%) developed rectal bleeding; in 9 patients (15\%) G3, Argon treatment was necessary (7 CT-based MUPIT [78\%] and 2 MRI-based TB [22\%]) at a median time of 8 months (7-14 months). Median $\mathrm{D}_{90}$ for the CTV was 74.4 Gy (range 69-78.8 Gy) for CT-based MUPIT and 80 Gy (range 79.6-80.4 Gy) for TB. Median rectum $\mathrm{D}_{2 \mathrm{~cm}^{3}}$ of these nine patients was $74.8 \mathrm{~Gy}$ (range 71.6-132.10 Gy) for CT-based MUPIT, and 71.3 Gy (69.972.7 Gy) for MRI-based TB. There were no bowel or bladder perforation events.

We analyzed the following factors using univariate analysis: stage (IIA vs. IIB vs. IIIA vs. IIIB vs. IVA), T (T2A vs. T2B vs. T3A vs. T3B vs. T4), nodal status (N0 vs. N1), vaginal involvement at diagnosis (disease free vs. involved), parametrial involvement at diagnosis (disease free vs. involved), myometrial involvement at diagnosis 
(yes vs. no), response to EBRT and chemotherapy (complete vs. partial), and CTV $\mathrm{D}_{90}(\leq 70 \mathrm{~Gy}$ vs. 70.05-75 Gy vs. 75-79 Gy vs. $>79.01 \mathrm{~Gy}$ ), rectum $\mathrm{D}_{2 \mathrm{~cm}^{3}}$ ( $\leq 75$ Gy vs. $>75.1$ Gy) for OS, DFS, LC, LNF, DM, and toxicity (rectal bleeding). All factors were analyzed alone and in relation to the type of applicator (MUPIT vs. TB). Of these, stage II, no lymph node involvement (N0), and no extension to the myometrium at diagnosis had a significant impact on OS and DFS $(p<0.001)$. None of prognostic factors analyzed influenced the local control.

There was sufficient follow-up in both groups to analyze the rate of development of rectal bleeding. A significant percentage $(p=0.040)$ of patients treated with CTbased MUPIT developed rectal bleeding. Twelve percent of patients treated with the MUPIT template developed G3 rectal bleeding. Three percent of those treated with TB developed rectal bleeding.

Due to the low number of other toxicities reported in follow-up, multivariate analysis was not done.

\section{Discussion}

In recent years, imaging has been incorporated into brachytherapy treatments. The replacement of $2 \mathrm{D}$ treatments based on point doses by 3D volumetric definitions has introduced a new era of gynecological BT [21]. The conventional "pear-shaped" homogeneous isodoses based on points included different volumes of tumor at different stages of the disease, and these were not routinely changed, based on response to EBRT. Smaller tumors and those with excellent and good responses were probably over-treated with large brachytherapy volumes; on the other hand, advanced stages with bigger tumors or poor response to EBRT may have been under-treated because of incomplete dosimetric coverage. Although definition of the cervix and the extension of carcinomas are less than optimal with $\mathrm{CT}$, this imaging modality has helped to assess morphological changes and brachytherapy volumes needing delineation. Today, MRI has been incorporated into brachytherapy for diagnosis and dosimetry because it provides superior definition of cervix tumors, their expansion, local involvement, and relationship with OARs. The new volume definitions possible with MRI have allowed the concept of IGBT to be defined by ABS and GECESTRO $[13,14,22,23]$ who have published several reports describing commissioning, reconstruction, and establishing MRI recommendations [24,25]. The purpose has been to facilitate the transition to MRI-based brachytherapy, incorporating "tailored" treatments with new applicators. Better results, increased local control, and decreased toxicity have been reported $[26,27,28,29,30,31,32,33]$ in on-going international protocols (EMBRACE, Retro EMBRACE, and EMBRACE II) that include hundreds of patients [34,35,36,37].

Magnetic resonance imaging has become the gold standard imaging technique for IGBT [20]. It has allowed identification of tumors that were not covered by standard intracavitary applicators (tandem and ovoids, or tandem and ring), and it has led to the development of new commercial devices that incorporate interstitial components. For instance, with the Utrecht and Vienna systems $[31,38]$, tumors with proximal and middle para- metrial involvement can be treated with a prescription isodose that can be increased by $15 \mathrm{~mm}$ laterally, compared to the coverage attainable with the classic point A approach [38]. Constraints can be maintained while achieving better dosimetric results than with a parametrial EBRT boost [39].

However, there are circumstances, in which these devices will prove insufficient for adequate treatment. For instance, in treating bulky tumors, cervix tumors that extend to the distal parametrium, tumors with medium or distal vaginal extension, those with poor response to EBRT, cases with anatomical constraints such as narrow vaginas or inability to enter the cervical OS, other interstitial approaches are needed $[19,40,41]$. Two commercial perineal interstitial templates (Syed-Neblett template or MUPIT template) are available $[9,10]$. And there are other published techniques such as adding transvaginal angled needles through new applicators (Vienna type 2) [42], the use of a complementary cap $[43,44]$, or an individual ring cap (3D impression) [45], insertion of additional "home-made" perineal needles or mono-institutional applicators (Nice applicator) [46,47]. In all cases, needles are guided free hand or by fluoroscopy, ultrasound, CT, or MRI [5,7,8,48,49].

We had used the MUPIT template for years, although the absence of an intrauterine component and need for CT for dosimetry with its associated artifacts produced by the metallic needles and its poor delimitation of tumor tissues proved inconvenient. In an effort to overcome these problems, in 2007 after starting using MRI for brachytherapy cervix dosimetry, we know that we needed an applicator that could combine intrauterine and vaginal components and interstitial needles, and that would include both straight and angled $\left(17^{\circ}\right)$ holes to cover distal parametrial disease, distal vagina disease, and bulky tumors. For all these reasons, in 2013, we have developed a new intracavitary/interstitial applicator compatible with MRI. The TB is an attempt to combine the technical advantages of MUPIT and the imaging advantages of MRI-based while preserving the stability, geometry, and robustness of the implant, covering the desired volume, avoiding possible errors of free-hand needle placement, and including an intrauterine component to provide a central brachytherapy dose. In this report, we analyze our clinical experience and results of 59 patients treated with CT-based MUPIT before April 2013 (34 patients), and with MRI-based TB and MRI dosimetry after this date (25 patients).

Both CT-based MUPIT and MRI-based TB groups had very similar baseline characteristics. MUPIT patients had more vaginal extension affectation, but the TB group included 3 patients with retroperitoneal and supraclavicular extension. There were no differences in survival, local control, lymph node control, or distant failure between the groups. The OS and DFS were worse at 12 and 24 months for TB patients. This is because the TB group included 2 patients with M1 disease with retroperitoneal lymph node involvement and two with more extensive IVA disease.

One notable difference between the two approaches was that the volumes defined in MRI-based TB were significantly smaller than those seen with CT-based MUPIT and its repercussion over toxicity. Overestimated CT-based volumes have been reported previously by Viswanathan 
et al. [18] in 10 patients with the applicator in place to decrease the potential for changes in the uterus and cervix, and there were no differences in OARs in the MRI planning. To eliminate the uncertainty of inter-observer target definition in our series, volumes for all patients were defined by the same experienced radiation oncologist [50,51]. The CTV volumes were smaller in patients treated with MRI-based TB (based on GEC-ESTRO volume definitions recommendations), even when they were compared by stage or extension. Because one potential criticism could be that the patients treated with MUPIT and TB were dissimilar, we performed CT scans (under the same conditions as MRI) in 7 patients implanted with TB. The data obtained from MRI showing better image definition, smaller CTV volumes, and smaller $\mathrm{D}_{90}$ over OARs are shown in Table 4.

In this study, there was no relationship between CTV volumes and local control, an outcome reported in other studies $[1,52,53]$. We take this as an evidence of the efficacy of perineal interstitial brachytherapy and its ability to adequately treat bulky disease, a result that has been reported by others [2].

Analysis of local recurrence in both groups showed that there were more myometrial recurrences in the CTbased MUPIT group. This was assumed to be due to the absence of an intrauterine component in these patients, with less dose reaching the myometrial extension and lack of a rectification of the organ. The use of a tandem is necessary to provide a higher central dose, and the absence of this dose is one of the disadvantages of the exclusive use of perineal interstitial templates. Viswanathan et al. have reported on this as significant predictor of survival [54].

The median $\mathrm{D}_{90}$ values of $75.8 \mathrm{~Gy}$ for CT-based MUPIT and 78.6 Gy for MRI-based TB are smaller than is recommended for increased local control $[55,56]$. These values should be interpreted with caution because they are related to the $\mathrm{CTV}_{\mathrm{IR}}$, and are associated with two different BT approaches: intracavitary alone and intracavitary with an interstitial component. We found that it was difficult to apply the GEC-ESTRO recommendations for doses to volumes of risk in this group of patients with locally advanced cervix carcinomas (patients with tumor extensions that were not covered by SIU/ovoids or the Utrecht applicator), primarily because this process involves a unique implant covering both diseases at the diagnostic $\left(\mathrm{CTV}_{\mathrm{IR}}\right)$, and disease at the time of the BT (GTV and $\left.\mathrm{CTV}_{\mathrm{HR}}\right)$. Due to the increased volume of the CTV, the total dose is administrated in smaller daily fractions, twice a day, over 4-6 days, according to Institutional protocol. As others have also reported, we prescribed the dose to this CTV without distinguishing between volumes and considering $\mathrm{CTV}_{\mathrm{HR}}$. What appears to be a relatively low median $\mathrm{EQD}_{2}$ to CTV was not taken into account like the effect of delivering 6 fractions, two times a day in a short overall treatment time, and our median $\mathrm{EQD}_{2}$ dose to CTV was within the range $\left(\mathrm{EQD}_{2} 61.6-82 \mathrm{~Gy}\right)$ described by others $[5,7,52,57,58]$.

Thus, although there are no guidelines for dose and fractionation schemes for a unique interstitial implant for locally advanced cervix carcinoma, we decided to continue administrating TB in six 4 Gy fractions, twice a day to the CTV, as we had done previously for CT-based MUPIT treatments, while following the GEC-ESTRO recommendations for tolerance doses of OARs. One possible disapproval of this approach is that the dose was the same in all cases, and that neither the size of the fractions nor the number of fractions was increased to account for residual disease at the time of the brachytherapy procedure.

Interstitial treatments with devices other than the Utrecht or Vienna applicators are not routinely used. Lack of experience, fear of complications, concern about the invasiveness of the procedure, and absence of randomized studies may be the reasons for persisting hesitance to use interstitial brachytherapy. Furthermore, most of the literature is based on limited numbers of patients and very heterogeneous clinical and dosimetry data: patients with cervix, vagina, or vulvar tumors with different biological behavior, primary and recurrences, with or without previous radiotherapy, treatments with CTV volumes ranging 63 to $137 \mathrm{~cm}^{3}$, different brachytherapy devices, and differing fraction sizes, total doses, dose specification methods, and optimization $[7,48,49,52,58,59,60]$. As a result, comparison or evaluation of resultant local control and survival is extremely difficult. In comparison with the results reported from the few studies of perineal interstitial brachytherapy for locally advanced cervix carcinoma, the outcomes of the current study are very similar in terms of OS, DFS, and LC $[2,5,8]$.

Comparison of toxicity is equally difficult. The published series define toxicity differently, use dissimilar grading systems, and vary in their approach to localization of the tumor, treatment volume size, cumulative dose received, and hot spots in the dosimetry. The most frequently reported interstitial implant complication is bleeding [4,61], and this is associated with factors such as tumor necrosis. In our series, only two patients $(3 \%)$ had bleeding after removal the applicator; this rate has been reported as high as $11 \%$ by others $[5,61]$. Bleeding risk is a potential event that has been correlated with individual heterogeneity in blood vessel architecture, tumor, and degree of necrosis of the tumor [47].

Published late complications vary and have no relationship to DVH. Rectum $\mathrm{D}_{2 \mathrm{~cm}^{3}}$ values are higher with interstitial brachytherapy, possibly because of the necessity of covering a larger region that is not possible with standard tandem implants. We have not found a correlation between dose histogram values and rectal toxicity as reported by D'Souza, Beriwal, Dyk, and Georg [1,49,58,62]. Unlike other authors [5,6,52], George et al. reported a $\mathrm{D}_{2 \mathrm{~cm}^{3}}$ $\left(\mathrm{EQD}_{2}\right.$ in rectum) that was $75 \mathrm{~Gy}$ for patients with late G2-G4 rectal complications. Lee et al. found that a median value of $73 \mathrm{~Gy}$ for $\mathrm{D}_{2 \mathrm{~cm}^{3}}$ in rectum was a strong predictor of late rectal G3-G4 complications. In our study, the median value for rectum $\mathrm{D}_{2 \mathrm{~cm}}$ exceeded $73 \mathrm{~Gy}$ in 37 patients (56\%) and 75 Gy in 29 patients (49\%), but only 4 developed G3 rectal bleeding. The majority of our patients with G3 toxicity were treated with CT-based MUPIT, and, in $92 \%$ of these patients, the entire vagina was treated, which explains the higher volumes of rectum included. Univariate analysis showed that the use of MUPIT was the unique variable with a direct relationship to rectal toxicity, probably because of the larger treatment volumes. 
Recently, Mazeron et al. [63] have published the dosevolume effect relationship for late rectal morbidity of 960 patients treated in EMBRACE protocol. This data has to be compared cautiously with patients treated exclusively with a perineal IC/interstitial implant, mainly because of the bigger volumes of treatment in the last ones. The first difference between their patients and our series is the technique employed. Only $34.4 \%$ of the patients of EMBRACE protocol were treated with interstitial BT (Utrecht applicator, Vienna applicator or free hand inserted needles). These is the reason of the very low percentage of rectitis (rectal bleeding), $16.3 \%$ of the patients (only 1\% G3) in comparison with our results (30\% rectal bleeding, $15 \%$ G3).

The tolerance doses recommended by GEC-ESTRO were exceeded in only 3 of the 9 patients with G3 rectal bleeding, and again all were treated with CT-based MUPIT (EQD $2 \mathrm{~cm}^{3}$ of rectum: 76.3 Gy, 80.2 Gy, and 132.10 Gy). Our overall 15\% rate of chronic G3 rectal bleeding is similar to that reported in the literature [58] and clearly reflects the difference between the applicators $(12 \%$ rate with CTbased MUPIT and 3\% with MRI-based TB).

Further studies of these applicators are needed to establish the best size for each fraction and the optimum number of fractions necessary to obtain the ideal $\mathrm{EQD}_{2}$ for complete coverage of the CTV, and to ascertain the appropriate doses to the $\mathrm{CTV}_{\mathrm{HR}}$ and the $\mathrm{CTV}_{\mathrm{IR}}$ to achieve local control and limit toxicity in this group of patients with aggressive disease and poor outcomes.

\section{Conclusions}

Magnetic resonance imaging-based planning has superseded CT for planning brachytherapy of cervix carcinoma. Interstitial brachytherapy can be employed in locally advanced cervix carcinomas when intracavitary techniques alone do not reach the residual tumor in areas of risk. The ability of MRI to better define the cervix, tumor, residual areas, and organs at risk allows smaller treatment volumes to be used, and smaller volumes have been shown to significantly decrease toxicity (3\% rectal bleeding G3). With a clearer understanding of the benefits of MRI-based planning, future studies are planned that will escalate doses in patients with a partial response defining CTVs following GEC-ESTRO recommendations. The TB offers an MRI compatible applicator that incorporates an endocavitary component with an interstitial one, thus ensuring central dose contribution. This device can be employed in different perineal diseases (primary or recurrences) including other gynecologic tumors as well as cervix.

\section{Acknowledgments}

The authors wish to thank Dr. Rosa Cañon for her help and involvement in the follow-up of the patients, and to Mr. David Carpenter for editorial assistance.

\section{Disclosure}

Authors report no conflict of interest.

\section{References}

1. Beriwal S, Bhatnagar A, Heron D et al. High-dose-rate interstitial brachytherapy for gynecologic malignancies. Brachytherapy 2006; 5: 218-222.

2. Pinn-Bingham M, Puthawala A, Syed N et al. Outcomes of high-dose-rate interstitial Brachytherapy in the treatment of locally advanced cervical cancer: long-term results. Int J Radiat Oncol Biol Phys 2013; 85: 714-720.

3. Syed A, Puthawala A, Abdelaziz N et al. Long term results of low-dose-rate interstitial-intracavitary brachytherapy in the treatment of carcinoma de cervix. Int J Radiat Oncol Biol Phys 2002; 54: 67-78.

4. Demanes D, Rodriguez R, Bendre D et al. High dose rate transperineal brachytherapy for cervical cancer. High pelvic control and low complications rates. Int J Radiat Oncol Biol Phys 1999; 45: 105-112.

5. Yoshida K, Yamazaki H, Takenaka T et al. A dose-volume analysis of magnetic resonance image-aided high dose rate image-based interstitial brachytherapy for uterine cervical cancer. Int J Radiat Oncol Biol Phys 2010; 77: 765-772.

6. Thibault I, Lavalle M, Aubin S et al. Inverse-planned gynecologic high dose rate interstitial brachytherapy: clinical outcomes and dose-volume histogram analysis. Brachytherapy 2012; 11: 181-191.

7. Viswanathan A, Szymonifka J, Tempary-Afdhal C et al. A prospective trial of real-time magnetic resonance-guided catheter placement in interstitial gynecologic brachytherapy. Brachytherapy 2013; 12: 240-247.

8. Yoshida K, Yamazaki H, Takenaka T et al. Preliminary results of MRI-assisted high dose rate interstitial brachytherapy for uterine cervical cancer. Brachytherapy 2015; 14: 1-8.

9. Martinez A, Cox R, Edmundson G. A multiple-site perineal applicator (MUPIT) for treatment of prostatic, anorectal and gynecologic malignancies. Int J Radiat Oncol Biol Phys 1984; 10: 297-305.

10. Syed A, Puthawala A, Neblett D et al. Transperineal interstitial intracavitary "Syed-Neblett" applicator in the treatment of carcinoma of the uterine cervix. Endocuriether Hypertherm 1986; 2: 1-13.

11. Villalba Rodríguez S, Sancho JR, Palacin Otal A et al. A new template for MRI based intracavitary/interstitial gynecologic brachytherapy: design and clinical implementation. J Contemp Brachytherapy 2015; 7: 265-272.

12. Richart J, Otal A, Rodriguez S et al. A practical MRI-based reconstruction method for a new endocavitary and interstitial gynaecological template. J Contemp Brachytherapy 2015; 7: 407-414.

13. Haie-Meder C, Pötter R, Van Limbergen E et al. Recommendations from Gynecological (GYN) GEC-ESTRO Working Group (I): concepts and terms in 3D image based 3D treatment planning in cervix cancer brachytherapy with emphasis on MRI assessment of GTV and CTV. Radiother Oncol 2005; 74: 235-245.

14. Pötter R, Haie-Meder C, Van Limbergen E et al. Recommendations from gynaecological (GYN) GEC ESTRO working group (II): concepts and terms in 3D image-based treatment planning in cervix cancer brachytherapy-3D dose volume parameters and aspects of 3D image-based anatomy, radiation physics, radiobiology. Radiother Oncol 2006; 78: 67-77.

15. Pecorelli S, Zigliani L, Odicino F. Revised FIGO staging for carcinoma of the cervix. Int J Gynaeco Obstet 2009; 105: 107-108.

16. Edge S, Byrd DR, Compton CC et al. (eds.). AJCC Cancer Staging Manual. Seventh Edition. Springer-Verlag, New York 2010.

17. Otal A, Richart J, Rodríguez S et al. Library development of Interstitial Gynecological Brachytherapy Utrecht and Benidorm 
Template to improve treatment planning reconstruction. World Congress of Brachytherapy. ABS-GEC ESTRO. San Francisco, 2016.

18. Viswanathan A, Dimopoulos J, Kirisits C et al. Computed tomography versus magnetic resonance image-based contouring in cervical cancer brachytherapy: results of a prospective trial and preliminary guidelines for standardized contours. Int J Radiat Oncol Biol Phys 2007; 68: 491-498.

19. Viswanathan A, Erickson B, Rownd J. Image guided approaches to interstitial brachytherapy. In: Viswanathan A, Kiristis C, Erickson B, Potter R (eds.). Gynecologic Radiation Therapy: Novel Approaches to Image-Guidance and Management. Springer, Berlin-Heidelberg 2011; pp. 247-259.

20. International Commission on Radiation units and Measurements. Prescribing, recording and reporting brachytherapy for cancer of the cervix. ICRU report 89. J ICRU 2016.

21. Harkenrider M, Alite F, Silva SR et al.; FACRO, FACR, FASTRO. Image-Based Brachytherapy for the Treatment of Cervical Cancer. Int J Radiat Oncol Biol Phys 2015; 92: 921-934.

22. Nag S. Controversies and new developments in gynecologic brachytherapy: image-based intracavitary brachytherapy for cervical carcinoma. Semin Radiat Oncol 2006; 16: 164-167.

23. Nag S, Cardenes H, Chang S et al. Image-Guided Brachytherapy Working Group. Proposed guidelines for image-based intracavitary brachytherapy for cervical carcinoma: report from Image-Guided Brachytherapy Working Group. Int J Radiat Oncol Biol Phys 2004; 60: 1160-1172.

24. Hellebust T, Kirisits C, Berger D et al. Recommendations from Gynaecological (GYN) GEC-ESTRO Working Group: Considerations and pitfalls in commissioning and applicator reconstruction in 3D image-based treatment planning of cervix cancer brachytherapy. Radiother Oncol 2010; 96: 153-160.

25. Dimopoulos J, Petrow P, Tanderup K et al. Recommendations from Gynecological (GYN) GEC-ESTRO Working group (IV): basic principles and parameters for MR imaging with the frame of image based adaptive cervix cancer brachytherapy. Radiother Oncol 2012; 103: 113-122.

26. Kang H, Shin K, Park S et al. 3D CT-based high dose rate brachytherapy for cervical cancer. Clinical impact on late rectal bleeding and local control. Radiother Oncol 2010; 97: 507-513.

27. Charra-Brunaud C, Harter V, Delanes M et al. Impact of 3D imaged-based PDR brachytherapy on outcome of patients treated of cervix carcinoma in France: results of the French STIC prospective study. Radiother Oncol 2012; 103: 305-313.

28. Pötter R, Dimopoulus J, Georg P et al. Clinical impact of MRI assisted dose volume adaptation and dose escalation in brachytherapy of locally advanced cervix cancer. Radiother Oncol 2007; 83: 148-155.

29. Pötter R, Georg P, Dimopoulos JC et al. Clinical outcome of protocol based image (MRI) guided adaptive brachytherapy combined with 3D conformal radiotherapy with or without chemotherapy in patients with locally advanced cervical cancer. Radiother Oncol 2011; 100: 116-123.

30. Lindegaard J, Fokdal L, Nielsen S et al. MRI-guided adaptive radiotherapy in locally advanced cervical cancer from a Nordic perspective. Acta Oncol 2013; 52: 1510-1519.

31. Nomden C, de Leeuw A, Moerland M et al. Clinical use of the Utrecht applicator for combined intracavitary/interstitial brachytherapy treatment in locally advanced cervical cancer. Int J Radiat Oncol Biol Phys 2012; 82: 1424-1430.

32. Rijkmans E, Nout R, Rutten I et al. Improved survival of patients with cervical cancer treated with image-guided brachytherapy compared with conventional brachytherapy. Gyn Oncol 2014; 135: 231-238.

33. Gill B, Kim H, Houser C et al. MRI-guided high dose rate intracavitary brachytherapy for treatment of cervical cancer:
The University of Pittsburgh experience. Int J Radiat Oncol Biol Phys 2015; 91: 540-547.

34. EMBRACE Protocol. Version 17-01-2008. Available at: https:// www. embracestudy.dk/UserUpload/PublicDocuments/EmbraceProtocol.pdf.

35. EMBRACE Study Committee. EMBRACE: An International Study on MRI-guided brachytherapy in locally advanced cervical cancer. Available at: https://www.embracestudy.dk/ About.aspx. Accessed January 5, 2015.

36. Sturdza A, Pötter R, Fokdal LU et al. Image guided brachytherapy in locally advanced cervical cancer: Improved pelvic control and survival in RetroEMBRACE, a multicenter cohort study. Radiother Oncol 2016; 120: 428-433.

37. Fokdal L, Sturdza A, Mazeron R et al. Image guided adaptive brachytherapy with combined intracavitary and interstitial technique improves the therapeutic ratio in locally advanced cervical cancer: Analysis from the retroEMBRACE study. Radiother Oncol 2016; 120: 434-440.

38. Kirisits C, Lang S, Dimopoulus J et al. The Vienna applicator for combined intracavitary and interstitial brachytherapy of cervical cancer: design, application, treatment planning and dosimetric results. Int I Radiat Oncol Biol Phys 2006; 65: 624-630.

39. Mohamed S, Kallehauge J, Fokdal L et al. Parametrial boosting in locally advanced cervical cancer: combined intracavitary/interstitial brachytherapy vs intracavitary brachytherapy plus external beam radiotherapy. Brachytherapy 2015; 14: 23-28.

40. Viswanathan A, Thomadsen B; American Brachytherapy Society Cervical Cancer Recommendations Committee; American Brachytherapy Society. American Brachytherapy Society consensus guidelines for locally advanced carcinoma of the cervix. Part I: General principles. Brachytherapy 2012; 11: 33-46.

41. Nag S, Erickson B, Thomadsen B et al. The American Brachytherapy Society recommendations for high dose rate brachytherapy for carcinoma of the cervix. Int J Radiat Oncol Biol Phys 2000; 48: 201-211.

42. Berger D, Potter R, Dimopoulos J et al. New Vienna applicator design for distal parametrium disease in cervical cancer. Brachytherapy 2010; 9: 551-552.

43. Petric P, Hudej R, Music M. MRI assisted cervix cancer brachytherapy pre-planning, based on the insertion of the applicator in para-cervical anesthesia: preliminary results of a prospective study. J Contemp Brachytherapy 2009; 1: 163-169.

44. Petric P, Hudej R, Hanuna O et al. MRI-assisted cervix cancer brachytherapy pre-planning based on application in paracervical anesthesia: final report. Radiol Oncol 2014; 48: 293-300.

45. Dimopoulus J. Interstitial techniques. Cervical cancer. ESTRO teaching course on image guiding radiotherapy \& chemotherapy in gynecological cancer - with and especial focus on adaptive brachytherapy. Utrecht, November 1-5, 2105.

46. Hannoun-Levi J, Chan-Fouche M, Gautier M et al. Interstitial preoperative high dose rate brachytherapy for early stage cervical cancer: dose-volume histogram parameters, pathologic response and early clinical outcome. Brachytherapy 2013; 12: 148-155.

47. Bailleux C, Tuan Falk A, Chand-Fouche $\mathrm{M}$ et al. Concomitant cervical and transperineal parametrial high dose rate brachytherapy boost for locally advanced cervical cancer. J Contemp Brachytherapy 2016; 8: 24-31.

48. Wang Y, Ye WJ, Du LH et al. Dose-volume parameters and clinical outcome of CT-guided free-hand high dose rate interstitial brachytherapy for cervical cancer. Chin J Cancer 2012; 31: 598-604.

49. Dyk P, Richardson S, Badiyan S et al. Outpatient-based highdose-rate interstitial brachytherapy for gynecologic malignancies. Brachytherapy 2015; 14: 231-237. 
50. Tanderup K, Nesvacil N, Potter R et al. Uncertain of target volume delineation in MRI guided adaptive brachytherapy of cervix cancer: a multi-institutional study. Radiother Oncol 2013; 107: 1-5.

51. Petrič P, Hudej R, Rogelj P et al. Uncertainties of target volume delineation in MRI guided adaptive brachytherapy of cervix cancer: a multi-institutional study. Radiother Oncol 2013; 107: 6-12.

52. Lee L, Damato A, Viswanathan A. Clinical outcomes of highdose-rate interstitial gynecologic brachytherapy using real time CT guidance. Brachytherapy 2013; 12: 303-310.

53. Lee L, Das I, Higgins $S$ et al. American Brachytherapy Society consensus guidelines for locally advanced carcinoma of the cervix. Part III: Low-dose-rate and pulsed-dose-rate brachytherapy. Brachytherapy 2012; 11: 53-57.

54. Viswanathan A, Cormark R, Rawal B et al. Increasing brachytherapy dose predicts survival for interstitial tandembased radiation for stage IIIB cervical cancer. Int J Gynecol Cancer 2009; 19: 1402-1406.

55. Dimopoulus J, Lang S, Kiristis C et al. Dose volume histogram parameters and local control tumor in magnetic resonance image guided cervical cancer brachytherapy. Int J Radiat Oncol Biol Phys 2009; 75: 56-63.

56. Kirisits C, Pötter R, Lang $S$ et al. Dose and volume parameters for MRI-based treatment planning in intracavitary brachytherapy for cervical cancers. Int J Radiat Oncol Biol Phys 2005; 62: 901-911.

57. Mahantshetty U, Shrivastava S, Kalyani N et al. Templatebased high dose rate interstitial brachytherapy in gynecologic cancers: A single Institutional experience. Brachytherapy 2014; 13: 337-342.

58. D'Souza D, Wiebe E, Sugimoto A et al. CT-based interstitial brachytherapy in advanced gynecologic malignancies: outcomes from a single institution experience. Brachytherapy 2014; 13: 225-232.

59. Sharma P, Sharma P, Swamidas J et al. Dose optimization in gynecological 3D image based interstitial brachytherapy using Martinez universal perineal template (MUPIT) - an institutional experience. J Med Phys 2014; 39: 197-202.

60. Isohashi F, Yoshiota Y, Koizumi M et al. High-dose-rate interstitial brachytherapy for previously untreated cervical carcinoma. Brachytherapy 2009; 8: 234-239.

61. Kuipers T, Hoekstra C, van't T et al. HDR brachytherapy applied to cervical carcinoma with moderate lateral expansion: modified principles of treatment. Radiother Oncol 2001; 58: 25-30.

62. Georg P, Lang S, Dimopoulos JC et al. Dose-volume histogram parameters and late side effects in magnetic resonance imageguided adaptive cervical cancer brachytherapy. Int J Radiat Oncol Biol Phys 2011; 79: 356-362.

63. Mazeron R, Fokdal L, Kirchheiner et al. Dose-volume effect relationships for late rectal morbidity in patients treated with chemoradiation and MRI-guided adaptive brachytherapy for locally advanced cervical cancer: Results from the prospective multicenter EMBRACE study. Radiother Oncol 2016; 120: $412-419$. 